\title{
Interfacial Structure and Structural Forces in Mixtures of Ionic Liquid with a Polar Solvent
}

\author{
Samuel W. Coles, ${ }^{1}$ Alexander M. Smith, ${ }^{1,2}$ Maxim V. \\ Fedorov, ${ }^{3,4}$ Florian Hausen, ${ }^{1,5}$ and Susan Perkin ${ }^{1, *}$ \\ ${ }^{1}$ Department of Chemistry, Physical and Theoretical Chemistry Laboratory, \\ University of Oxford, Oxford OX1 3QZ, U.K. \\ ${ }^{2}$ Department of Inorganic and Analytical Chemistry, \\ University of Geneva, 1205 Geneva, Switzerland \\ ${ }^{3}$ Department of Physics, Scottish Universities Physics Alliance (SUPA), \\ Strathclyde University, John Anderson Building, \\ 107 Rottenrow East, Glasgow G4 ONG, United Kingdom. \\ ${ }^{4}$ Skolkovo Institute of Science and Technology, \\ Skolkovo Innovation Center, Moscow 143026 Russia \\ ${ }^{5}$ Institute of Energy and Climate Research, IEK-9, \\ Forschungszentrum Jülich, 52425 Jülich and RWTH Aachen University, \\ Institute of Physical Chemistry, 52074 Aachen, Germany
}

Many applications of ionic liquids involve their mixtures with neutral molecular solvents. The chemical physics of these high-concentration electrolytes, in particular at interfaces, still holds many challenges. In this contribution we begin to unravel the relationship between measurements of structural ('solvation') forces in mixtures of ionic liquid with polar solvent and the corresponding structure determined by molecular dynamic simulations of the same mixtures. In order to make the quantitiative link between experiments with mica surfaces and simulations with fixed-charge surfaces, we present an experimental procedure for determining the effective surface charge on mica in ionic liquid. We find that a structural cross-over recently inferred from force measurement appears to be supported by simulation: At the cross-over, charge-oscillatory structure switches to charge-monotonic, and solvent layering becomes dominant. Finally, we map out a phase diagram in compositionsurface charge space delineating regions of charge-oscillatory interfacial structure and 
regions of charge-monotonic decay. We note that these features of structure and oscillatory forces are distinct from (acting simultaneously with) the recently reported longer range monotonic forces arising from anomalously long bulk screening lengths in high-concentration electrolytes.

\section{INTRODUCTION}

Mixtures of ionic liquid with molecular solvent are both of fundamental interest in the chemical physics of liquids and of great practical importance. The latter is manifest, whereas the former perhaps needs introduction given the great extant literature on electrolyte solutions. To do so we begin by recalling the comment of Robinson \& Stokes, when considering the influence of an ion on surrounding water molecules in highly concentrated electrolytes[1]: "In very dilute solutions it is permissible to think of the effects produced by a single ion on successive layers of water molecules, but in more concentrated solutions one meets the difficulty that 'the further from England the nearer is to France' ". Indeed, in a solution of 1:1 electrolyte at $2 \mathrm{M}$ concentration the average interionic distance is only $0.75 \mathrm{~nm}$; ions are typically separated by 1-2 water molecules. This evocative comment reminds us of impossibility of considering one component as the 'solvent' and the other 'solute', with the concomitant understanding that one is in large excess over the other, in electrolytes of concentration above about $1 \mathrm{M}$ or so. The simplifying assumptions employed in theories of dilute solutions, such as the Debye-Hückel theory, do not apply and we must find alternative frameworks to explain interfacial structure, molecular and surface interactions in concentrated electrolytes.

Recent interest in concentrated electrolytes has arisen from several intriguing physical properties qualitatively distinct from those of dilute electrolytes or ionic liquids. For example mixtures of water and a lithium salt at concentrations above $5 \mathrm{M}$ salt, at which point water is outnumbered by salt (in both weight and volume), lead to a doubling of the electrochemical window compared to dilute aqueous electrolytes and these have been used to demonstrate a "water-in-salt" lithium ion battery[2]. Conductivity of electrolytes is also a critically important parameter for device performance, and this can also be tuned (maximised) by doping ionic liquids or salts with relatively small quantities of solvent[3]. Maximal

\footnotetext{
* susan.perkin@chem.ox.ac.uk
} 
conductivity occurs at a "sweet spot" where viscosity is sufficiently reduced by the lubricating effect of solvent between the ions, and where the number density of free charge carries is still sufficiently high.

Electrochemical and nanotechnological applications of ionic liquid-solvent mixtures pivot on the solid-liquid interfacial structure and properties. The structure in pure ionic liquids adjacent to charged surfaces has been the focus of much scrutiny over the past decade, and the oscillating layers of excess cation and anion are now relatively well characterised[4]. At the opposite end of the concentration spectrum - when ions are diluted to $<\mathrm{mM}$ concentration in a polar solvent such as water - the resulting electrical double layer structure is of course well described by classical Stern and Gouy-Chapmann models[5]. Only a few recent experimental[6-10] and simulation[11, 12] studies have focussed on the intermediate concentration regime where neither ions nor solvent are in great excess.

The aim of the present work is to obtain insight into the near-surface structure and structural forces in mixtures of ionic liquid with a polar solvent (propylene carbonate). This will be achieved by comparison between a molecular dynamics (MD) simulations of the mixtures at a charged surface with experimental surface force balance (SFB) measurements in similar systems. The MD simulations reveal aspects of the molecular structure in the liquid at a charged interface, and how this varies with electrolyte concentration and surface charge. We will address the following questions: (i) In what way does the presence of solvent modify or disrupt the arrangement of ionic liquid ions at charged surfaces, compared to the case of pure ionic liquid? (ii) What is the relationship between force between charged surfaces - as measured in SFB and AFM experiments - and molecular structure at the interface (from simulation)? (iii) What is the surface charge on mica in ionic liquids, and how does surface charge relate to the structure in adjacent electrolyte?

To facilitate discussion of these questions, we will frame our arguments around the parameters of the following equation, which is the simplest empirical expression which approximately represents the force between mica surfaces across concentrated electrolytes:

$$
F_{N} / R=A e^{-D / \lambda_{o}} \cos (\omega D+\phi)+B e^{-D / \lambda_{s}} .
$$

In Equation (1) $F_{N}$ is the force measured between crossed cylinders (or sphere and flat), curvature radius $R$, at closest separation distance $D$. Typically, $F_{N}, R, D$ are measured directly whilst $\lambda_{o}, \lambda_{s}, \omega, A$ and $B$ are fitting paramters. Examples of typical measurements 
and the fitted function are shown in Figure $1 \mathrm{~B}$ and $\mathrm{C}$. The damped oscillatory component of the force in Equation (1) has been interpreted qualitatively in the past[13]; the oscillations have been rationalised in terms of expulsion of layers of ionic liquids from between charged surfaces and the details rationalised in terms of influence of different molecular characteristics on the measured force. However there has been only little quantitative interpretation of the magnitude, $A$, and decay, $\lambda_{o}[14,15]$. Recently, some of us have shown that the wavelength of the damped decay, $2 \pi / \omega$, undergoes a switch when solvent is added to an ionic liquid, from a ionic liquid-determined wavelength at high concentration to a solvent-determined wavelength at approximately $35 \mathrm{~mol} \%$ ionic liquid[10].

The second term on the right of Equation (1) represents the longer ranged monotonic component observed in force measurements across concentrated electrolytes[16-19]. We have written about this long-range force in ionic liquids and concentrated electrolytes and the scaling of the screening length $\lambda_{s}$ elsewhere recently[17, 20]; it will not be the subject of this paper. Nonetheless it is important to note that the decay of the oscillatory structural force, $\lambda_{o}$, is distinct from the screening length, $\lambda_{s}$, and they each scale differently with concentration[10]. The presence of a longer-range force with a decay length up to $\lambda_{s}=$ $10 \mathrm{~nm}$ implies that the oscillatory structural component at short range - the first term on the right of Equation (1) - is not the asymptotic component of the surface force and is therefore not expected to be captured by asymptotic analysis of the correlation functions e.g. using the Mean Spherical Approximation as in [21].

Whilst $\lambda_{s}$ is a property of the bulk electrolyte, $A$ and $B$ are expected to be surfacedependent. The wavelength and decay of the oscillatory component, described by $2 \pi / \omega$ and $\lambda_{o}$, appear to be determined by both liquid and surface characteristics and it is this latter interplay that we explore here. We will be principally concerned with the inspection of ion density distributions (from MD simulation) in order to guide interpretation of the parameters $A, \lambda_{o}$ and $\omega$ obtained from force measurement. Importantly, we present an experiment to determine the surface charge of mica in one ionic liquid in order to provide a point of comparison between the force measurements and the simulations with varying surface charge. Thus, with experimental measurements of surface forces and molecular dynamics simulations of interfacial structure, we investigate how liquid composition (dilution of ionic liquid with solvent) and surface charge impact the short-range oscillatory structure at electrolyte-electrode surfaces. 


\section{METHODS}

Experimental measurement of forces between crossed-cylinder surfaces across the liquid mixtures were performed using the surface force balance (SFB). The experimental details are largely the same as described previously[22][10]. Atomically smooth mica sheets (0.3$1.0 \mathrm{~cm}^{2}$ area and $1-3 \mu \mathrm{m}$ thick) were back-silvered before transferring onto hemi-cylindrical lenses and mounted in crossed orientation such that the relative geometry is similar to a sphere approaching a flat plate. The resulting silver-mica-liquid-mica-silver stack acts as an interferometric cavity. Bright columnated white light incident on interferometer, dispersed with a spectrometer, emerges as a set of bright fringes of equal chromatic order (FECO). The bottom lens is mounted on a horizontal leaf spring, while the top lens is mounted on a piezo-electric tube (PZT). By expanding the PZT, the top surface is brought at constant velocity towards the bottom surface from separations $D$ of $200-400 \mathrm{~nm}$ to $D$ of one or a few molecular diameters. $D$ and $F_{N}(D)$ are calculated from the FECO spectrum, with points taken at a rate of approximately $10 \mathrm{~s}^{-1}$.

In experiments where the opposing substrates were mica and gold, smooth gold surfaces were prepared by diffusion bonding two $30 \mathrm{~nm}$ gold films at room temperature over several days, and template stripping from mica as first described by Parker and Christenson for silver surfaces[23]. The resultant $60 \mathrm{~nm}$ gold film was then glued onto a cylindrical lens mica side down to serve as a working electrode, and an insulated connecting wire was attached using conductive epoxy. The same gold film acts as both the mirror for FECO interferometry and smooth potential-controlled electrode surface for the force measurement. Two platinum wires were used as quasi reference and counter electrodes. Prior to immersion in the ionic liquids, the platinum electrodes were rinsed with water and ethanol, dried, and passed through a flame to remove organic contaminants. Electrochemical control and measurement was with an Metrohm Autolab PGSTAT128N potentiostat.

The ionic liquids used were 1-butyl-1-methylpyrrolidinium bis[(trifluoromethane)sulfonyl]imide, $\left[\mathrm{C}_{4} \mathrm{C}_{1} \mathrm{Pyrr}\right]\left[\mathrm{NTf}_{2}\right]$ (Iolitec, 99\%), and 1-ethyl-3-methylimidazolium bis[(trifluoromethane)sulfonyl]imide, $\left[\mathrm{C}_{2} \mathrm{C}_{1} \mathrm{Im}\right]\left[\mathrm{NTf}_{2}\right]$ (Iolitec, 99.5\%). Liquids were dried in vacuo $\left(10^{-2} \mathrm{mbar}, 70^{\circ} \mathrm{C}\right)$ overnight before preparing the electrolyte solutions. Propylene carbonate (Sigma Aldrich, anhydrous, 99.7\% was used as received from freshly opened bottles. A droplet of solution of approximately $50 \mu \mathrm{L}$ was injected between the lenses which is sufficient to create a large reservoir 
of bulk solution outside of the confined film.

Molecular dynamics simulations were carried out using the Gromacs 5.1.4 package[24, 25]. The simulation method is similar to that used in previous studies [26][27]. The ionic liquid is modelled using the OPLS-AA[28] based CL\&P[29, 30] force field with the relative permittivity set to 1.6 to account for the effect of polarisability. The propylene carbonate molecules are modeled using the OPLS-AA force field[28] with slight reparameterisation to obtain an accurate dielectric constant[31, 32]. Details of the force fields are provided in the supplementary information. The simulation system consists of two graphene like slab electrodes with liquid in between. The number of ion pairs of the confined ionic liquid mixture is selected in order for the liquid to be at atmospheric density (the number of ion pairs for each of the liquids is listed in Table I).

TABLE I. Table showing the composition of simulation boxes studied.

\begin{tabular}{|l|l|l|l|}
\hline$X_{\mathrm{IL}}$ & $N_{\text {cations }}$ & $N_{\text {anions }}$ & $N_{\mathrm{PC}}$ \\
\hline 1 & 300 & 300 & 0 \\
\hline 0.8 & 284 & 284 & 71 \\
\hline 0.6 & 256 & 256 & 169 \\
\hline 0.5 & 238 & 238 & 238 \\
\hline 0.4 & 214 & 214 & 321 \\
\hline 0.2 & 143 & 143 & 572 \\
\hline
\end{tabular}

In the simulations the capacitor electrodes are modelled as graphene like slabs consisting of discrete carbon atoms in a graphene like arrangement. The carbon atoms within the graphene are frozen in space throughout the simulation. The gap between the graphenes is set to be $12.6410 \mathrm{~nm}$, while the $\mathrm{x}$ and $\mathrm{y}$ dimensions of the periodic simulation cell $3.4080 \mathrm{~nm}$ by $3.4433 \mathrm{~nm}$. The procedure for the simulations is as follows. Ions are packed into boxes using the packmol algorithm. This is followed by steepest decent minimisation of the liquid slabs. Once minimisation has taken place equal and opposite charges are applied to the two electrodes. Replicas are generated for surface charges ranging from $-37 \mu \mathrm{C} / \mathrm{cm}^{-2}$ to $+37 \mu \mathrm{C} / \mathrm{cm}^{-2}$ with equal and opposite charge being placed on the two electrodes in each replica. Each replica was then annealed from $300 \mathrm{~K}$ to $500 \mathrm{~K}$ and back over the course of $2 \mathrm{~ns}$ followed by a further $1 \mathrm{~ns}$ of equilibration at $300 \mathrm{~K}$. A production run of $2 \mathrm{~ns}$ at $300 \mathrm{~K}$ 
is then performed. This process is repeated for each liquid mixture for each surface charge 2 additional replicas. Time steps for all dynamical steps are set as $1 \mathrm{fs}$. The simulations described were performed under $N V T$ conditions, with temperature conditions maintained using the V-rescale thermostat.[25, 33-35] The Coulomb and van der Waals cut-offs were set to $1 \mathrm{~nm}$. Long-range electrostatics were performed using the particle mesh Ewald method, with a slab geometry[36, 37].

\section{THE STRUCTURAL TRANSITION OBSERVED IN FORCE MEASUREMENTS BETWEEN MICA SURFACES ACROSS MIXTURES OF IONIC LIQUID AND PROPYLENE CARBONATE}

In a recent series of experiments[10] some of us investigated the strucutral forces between mica sheets across mixtures of 1-butyl-1-methylpyrrolidinium bis[(trifluoromethane)sulfonyl] imide, $\left[\mathrm{C}_{4} \mathrm{C}_{1} \mathrm{Pyrr}\right]\left[\mathrm{NTf}_{2}\right]$, with the polar solvent propylene carbonate. This mixture was chosen because of the miscibility of the ionic liquid and solvent in all proportions at room temperature. The oscillatory structural force arising from squeeze-out of propylene carbonate between two planar mica surfaces is already known[38], as is the structural force in the pure ionic liquid[39]. Our interest was to discover the nature of structural forces at intermediate concentrations. Which oscillatory wavelength, solvent or salt, 'wins out'? Would there be any structure apparent at all?

It was found that indeed oscillatory solvation forces are measured at all concentrations studied, and - most notably - that there is a sharp switch in the wavelength of oscillatory force, $2 \pi / \omega$, between $2.0-2.5 \mathrm{M}$ (or between $30-45 \mathrm{~mol} \%$ salt). Below this cross-over point $2 \pi / \omega$ is static at the solvent-determined value of $0.55 \mathrm{~nm}$, whereas above the cross-over concentration $2 \pi / \omega=0.80 \mathrm{~nm}$. Figure 1 shows a summary of this finding: in panel A the wavelength $2 \pi / \omega$ is plotted for concentrations ranging from 0-100 \% salt, and below are examples of the raw force profles and fitted curves according to Equation (1).

The magnitude, $A$, and decay length, $\lambda_{o}$, also vary systematically with concentration[10]: The decay length of the oscillatory envelope enclosing the oscillatory force, $\lambda_{o}$, appears to be intimately connected with the wavelength and undergoes a similar transition. $\lambda_{o} \sim 0.85 \pm$ $0.30 \mathrm{~nm}$ at low concentrations below the transition, and $\lambda_{o} \sim 1.4 \pm 0.4 \mathrm{~nm}$ at concentrations above the transition. The pre-factor determining the magnitude of oscillatory solvation 


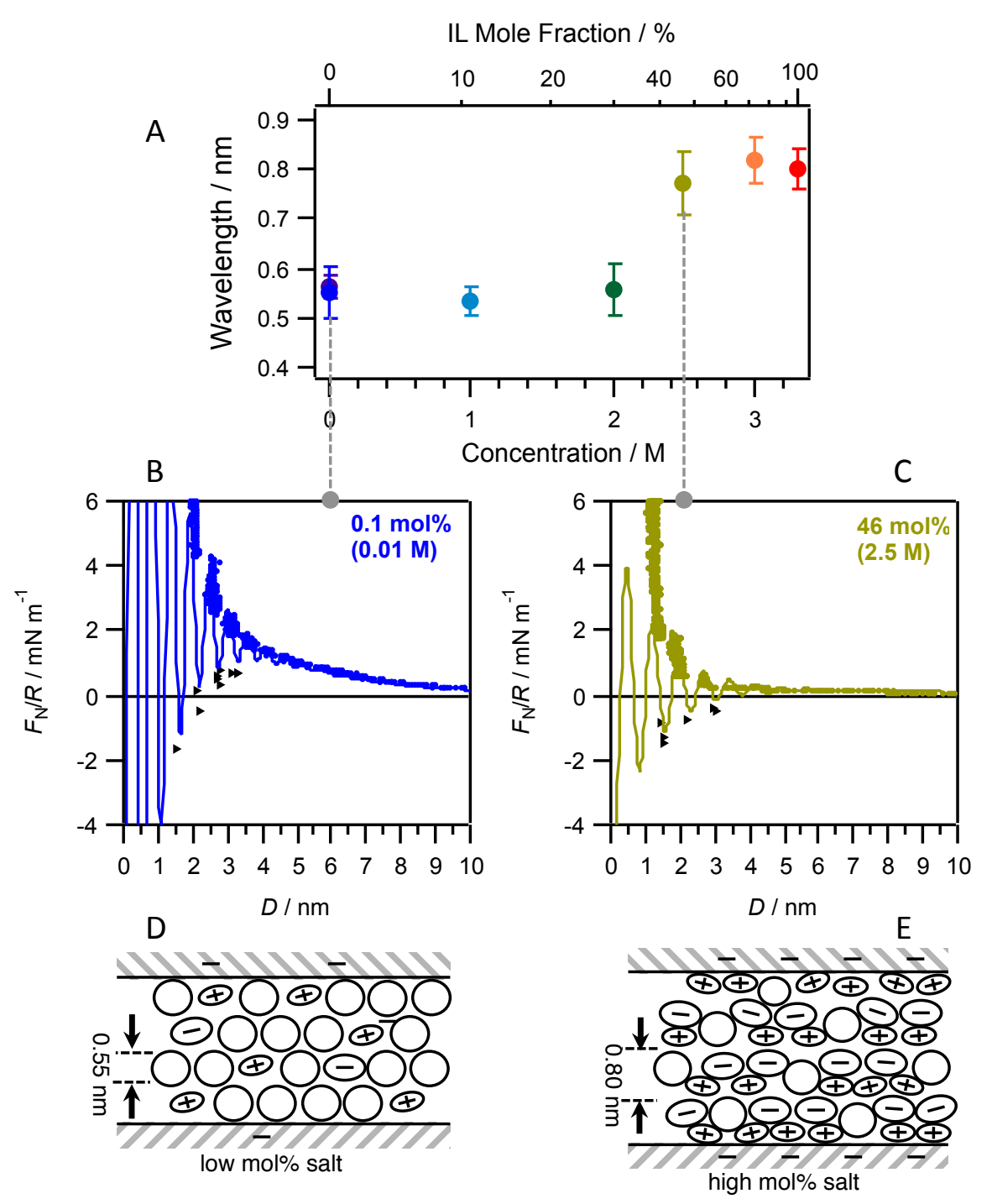

FIG. 1. A: Experimental measurements of the wavelength of the oscillatory structural force between two mica surfaces across mixtures of $\left[\mathrm{C}_{4} \mathrm{C}_{1} \mathrm{Pyrr}\right]\left[\mathrm{NTf}_{2}\right]$ and propylene carbonate at various mole fractions. The wavelength, $\lambda_{o}$, values are extracted from fits of the full measured force profile to Equation (1); examples of the force profiles (circular points measured on compression and triangles on decompression) and fits (solid lines) are shown in panels B and C. We emphasise the importance of using both 'in' (compression) and 'out' (decompression) profiles for fitting of the SFB data. B shows the case of large excess of propylene carbonate (0.01M ionic liquid), whereas $\mathrm{C}$ shows an example at higher concentration of ionic liquid (2.5 M ionic liquid). These examples straddle the cross-over concentration, and the schematics below in D and E indicate our interpretation in terms of solvent-dominated layering at low conentration (D) and salt-determined layering at high concentration (E). Partially replotted from ref.[10] 
force, $A$ in Equation (1), shows maxima for the pure ionic liquid and for the pure solvent, and a minima in between at the point of the structural transition.

This was interpreted in terms of ions disrupting the solvent structure (at low concentration), and solvent disrupting the structure of ionic liquid (at high concentration). The drawings in Figure $1 \mathrm{D}$ and $\mathrm{E}$ interpret the switch in wavelength: at low concentration of salt, ions pack within the propylene carbonate layers, perhaps disrupting their order but not modifying the wavelength. At high concentration, on the other hand, propylene carbonate molecules sit within the salt layers as was inferred by Mezger from x-ray experiments[8].

It was proposed that the oscillatory structural forces arise from steric packing effects[10]; the observed switch in correlation length is reminiscent of the crossover predicted[40] and observed[41][42] for packing of big and small particles. To obtain greater insight into this interpretation we compare (in Sections V and VI) these measurements to the MD simulations of the same ionic liquid and polar solvent mixture.

\section{ON THE SURFACE CHARGE OF MICA IN IONIC LIQUID}

In order to compare quantitatively the liquid structure at a charged surface in an experiment to that in a simulation it is necessary to know the magnitude of the surface charge in the experiment. There has been much interest in the nature of ionisation of mica in ionic liquids, but to our knowledge no direct determination of its extent. Here we discuss the nature of the cleaved mica surface, its ionisation when immersed in polar liquids or electrolytes, and a new experiment to estimate the magnitude of surface charge on mica in an ionic liquid.

Upon cleavage of mica along its basal plane in air, potassium is randomly but evenly distributed between the two surfaces in order to neutralise the negative charge of each mica surface which arises from statistical substitution of aluminium for silicon[43]. This exposes high energy but overall neutral surfaces, with locally positive charge at potassium domains and locally negative charge in regions between with the physical cleaving process determining the mesoscopic domain arrangement. In solutions with sufficiently high dielectric constants, it is commonly known that potassium ions in mica dissociate, leaving a net negative charge. However, it is unclear to what extent this occurs for ionic liquids, which have moderately low dielectric constants. In a study comparing x-ray reflectivity data and atomistic molecular 
dynamics simulations for a mica-ionic liquid interface[44], the best quantitative agreement in charge density profiles for the two methods was achieved by removing all surface potassium ions in the simulations, resulting in the maximal surface charge density of $-32 \mu \mathrm{Ccm}^{-2}$. However we note that in those x-ray experiments the mica was first immersed in a centrifuge tube containing deionized water for more than one hour prior to drying and transferring to the sample cell, and this is certain to have a profound influence. Water has been shown to have an influence on the nanostructure of confined ionic liquids[45-48], but its effect on potassium desorption and resultant mica surface charge density is less clear. It has been inferred from force spectroscopy measurements that potassium desorbs from the mica surface only in the presence of wet ionic liquids[47], but this is in contradiction with recent AFM images which suggest substantial desorption in contact with a dry protic ionic liquid[49]. A pertinent related study on freshly cleaved mica surfaces in ambient conditions revealed $0.1 \mathrm{~nm}$ steps which subsequently disappeared in a matter of minutes[50]. The most likely explanation attributes the features to domains of potassium ions which become mobile upon sufficient adsorption of water from the ambient conditions. Hence it is clear that even for a completely dry ionic liquid, potassium desorption may be inevitable if the surfaces are exposed to ambient air prior to immersion in ionic liquid. Such a mechanism of hydrated potassium desorption may explain dewetting of ionic liquids on mica in UHV conditions[51] or after heating the surface[52], where there is little to no adsorbed water. In cases where water is present at the interface the solid-liquid interfacial tension is likely to be affected[53].

There is a measurable repulsive force between mica surfaces across a large variety of ionic liquids[45][16][17], indicating that they are indeed charged and potassium dissociation is occurring to some extent, however the link between the magnitude of repulsive force and the surface charge (and plane at which that charge is defined) is not clear in those experiments. Note that it is not appropriate to apply a DLVO framework to the analysis of ionic liquids and concentrated electrolytes due to lack of accurate description of packing effects or correlations[54], and so it is not possible to extract the surface charge density by substituting the measured decay length into the Poisson-Boltzmann equation. Nevertheless, the surface charge density must lie in the range $-32<\sigma<0 \mu \mathrm{Ccm}^{-2}$ - the maximal magnitude arising if all potassium ions were dissociated - and the amplitude of the longrange electrostatic forces must be in some way related to the mica surface charge density. Therefore we suggest a crude estimate by comparing with capacitance measurements as 


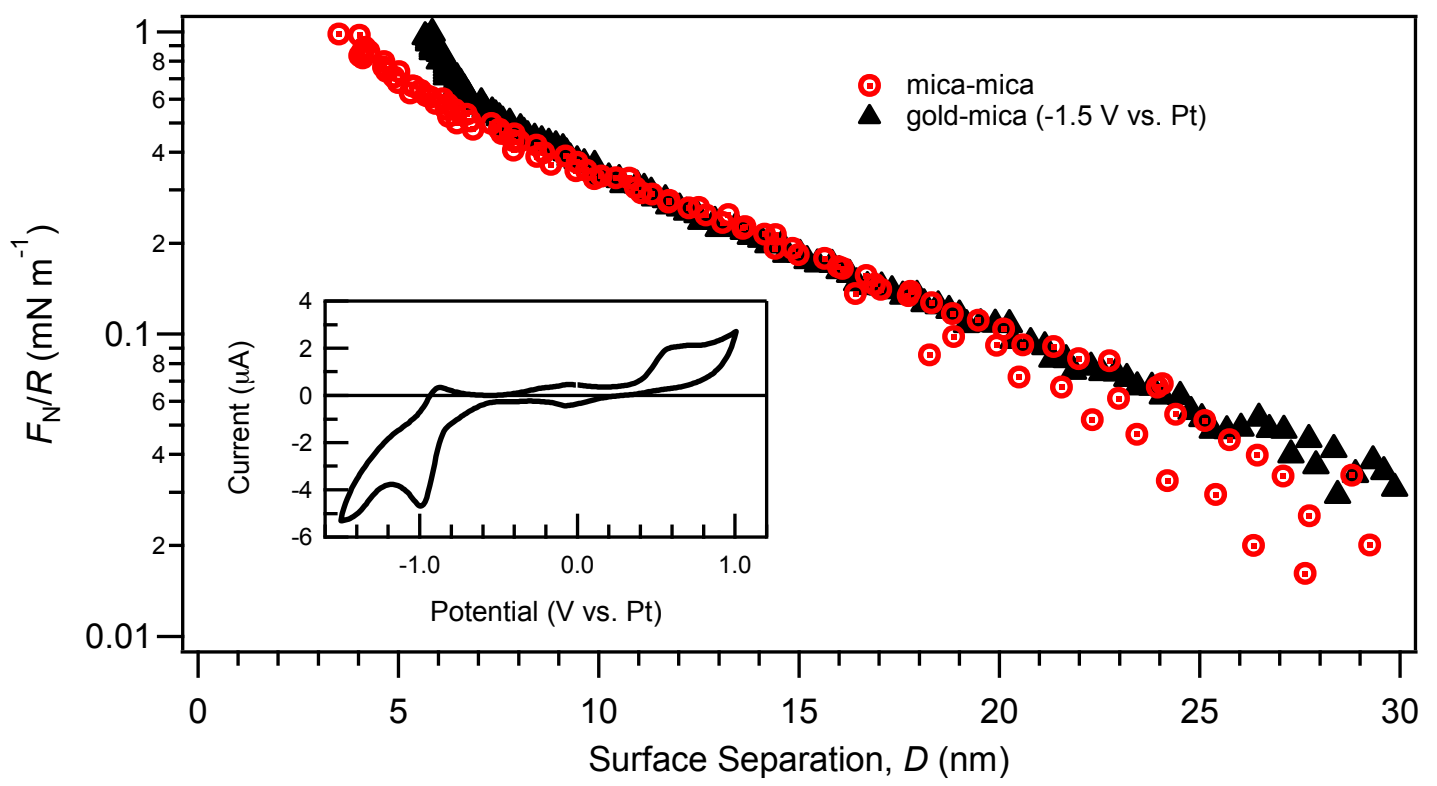

FIG. 2. Measured forces (normalised by radius of curvature, $R$ ) as a function of surface separation, $D$, between two mica surfaces (red circles) and between one mica surface and one gold surface with an applied potential of $-1.5 \mathrm{~V}$ vs. Pt (black triangles) across the ionic liquid $\left[\mathrm{C}_{2} \mathrm{C}_{1} \mathrm{Im}\right]\left[\mathrm{NTf}_{2}\right]$. The inset shows a cyclic voltammogram for the template stripped gold surface used in the experiment.

follows.

We measured the force between a mica surface and a template-stripped (smooth) gold surface across a pure imidazolium-based ionic liquid, and observed the variation in the longrange component of the force when the potential applied to the gold surface was varied. Variation in the gold potential led to variation in the magnitude of the long-range force, and thus we were able to determine the potential which must be applied to the gold surface in order that the long-range electrostatic force has a matching magnitude to that measured between two mica surfaces across the same liquid. Figure 2 shows this occurs at an applied potential of $-1.5 \mathrm{~V}$ vs. $\mathrm{Pt}$ for the ionic liquid $\left[\mathrm{C}_{2} \mathrm{C}_{1} \mathrm{Im}\right]\left[\mathrm{NTf}_{2}\right]$. Then, by comparing to capacitance-potential curves for the same ionic liquid at gold electrodes[55][56], we estimate the surface charge density of gold to be approximately $-20 \mu \mathrm{Ccm}^{-2}$ at this applied potential. We note that surface roughness effects on gold and the nature of discrete charge sites on mica leads to difference in the arrangement of near-surface ions between mica and gold, and this is likely to alter the screening and capacitance properties of the two different interfaces. Thus our assumption that similar surface charge densities for the two surfaces will result in 
similar double layer forces is a rather crude one; nonetheless this simple estimate seems to give a feasible result which is of use when comparing experiments to computer simulations where a fixed surface charge (or potential) must be selected as an input. A similar method was found to work well in the case of dilute electrolytes and similar gold/mica SFB setup in earlier work of Tivony et al.[57]. As a result of this analysis, we suggest that the most direct comparison for experimental results with mica surfaces in this pure ionic liquid is to simulations with a fixed surface charge density of $-20 \mu \mathrm{Ccm}^{-2}$. We note that the presence of solvent and different chemical groups on the ionic liquid will of course alter the effective surface charge on mica; a more complete survey of the surface charge of mica - determined according to the procedure above and exploring these various parameters - is beyond the scope of this paper but would be of use in the future.

\section{MOLECULAR DYNAMIC SIMULATIONS OF THE INTERFACE BETWEEN AN ELECTRODE AND IONIC LIQUID : PROPYLENE CARBONATE LIQUID MIXTURES}

We performed molecular dynamic simulations of $\left[\mathrm{C}_{4} \mathrm{C}_{1} \mathrm{Pyrr}\right]\left[\mathrm{NTf}_{2}\right]$ and propylene carbonate mixtures in contact with a fixed-charge surface, systematically varying the mole fraction of ionic liquid in propylene carbonate and the charge density on the surface. In Figure 3 we present a summary of the resulting nanostructure of the liquid mixture in the region $0.0-2.5 \mathrm{~nm}$ from the charged surface (which is located at $0.0 \mathrm{~nm}$ ). The density plots on the left hand side of Figure 3 show the local charge density as a function of distance from the electrode and as a function of surface charge, i.e. $\rho_{\Delta}=\rho_{+}-\rho_{-}$where $\rho_{+}$is the local number density of cations and $\rho_{-}$is the local number density of anions. Red (blue) regions therefore indicate excess cation (anion) density. Each plot, A-F, corresponds to a different mole fraction of ionic liquid ranging from $x_{I L}=100 \mathrm{~mol} \%$ (in A) to $x_{I L}=20 \mathrm{~mol} \%$ (in F).

On the right hand side of Figure 3 we provide, for each mole fraction, the density profile of cations (red), anions (blue), and solvent (green) at two distinct values of surface charge $\left(-10 \mu \mathrm{Ccm}^{-2}\right.$ and $\left.-20 \mu \mathrm{Ccm}^{-2}\right)$. As outlined in the previous section, the density profiles at $-20 \mu \mathrm{Ccm}^{-2}$ are expected to be most directly comparable to SFB experiments with mica surfaces.

Inspecting first the density plot and profiles for pure ionic liquid, Figure $3 \mathrm{~A}(\mathrm{i}-\mathrm{iii})$, it is 

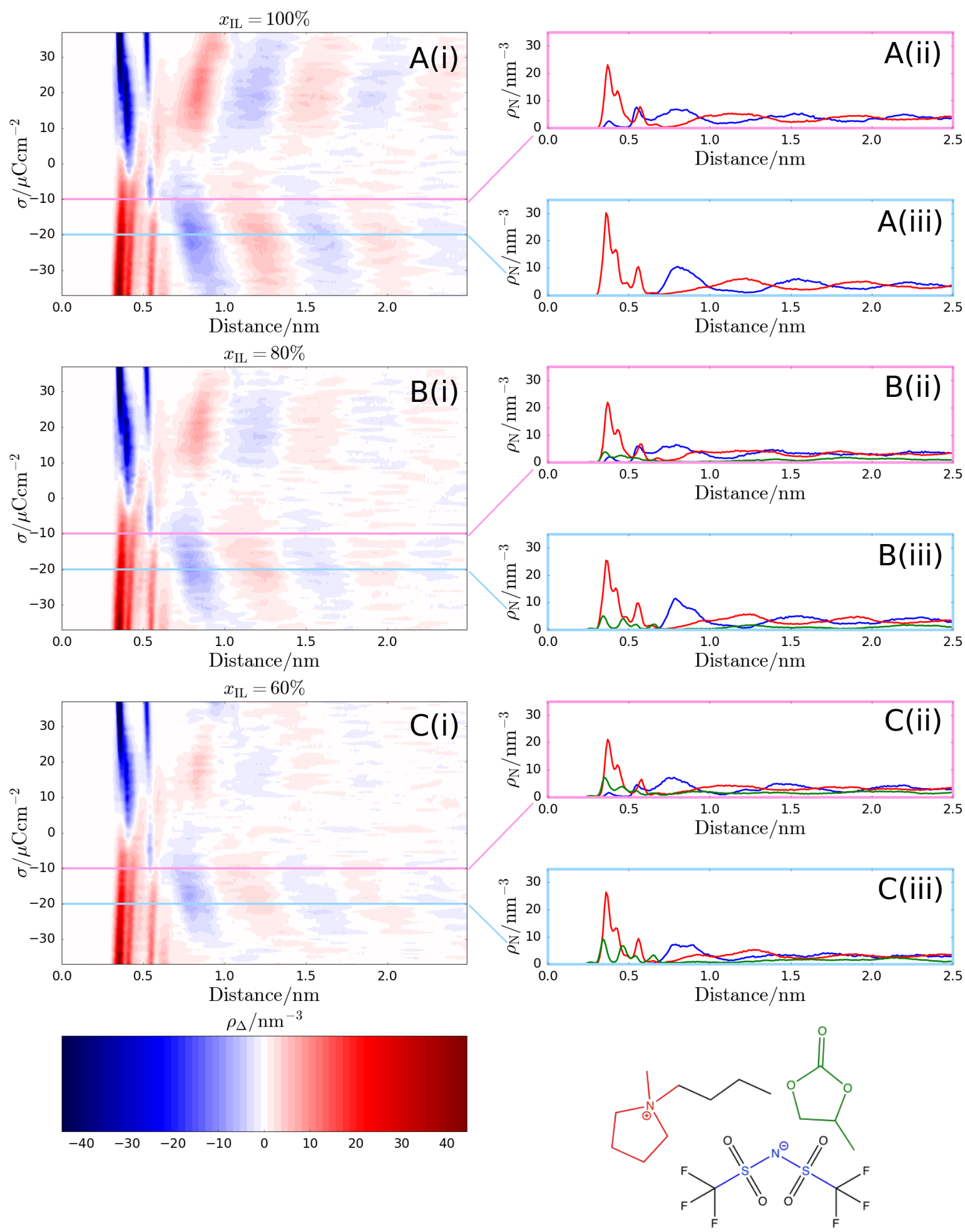

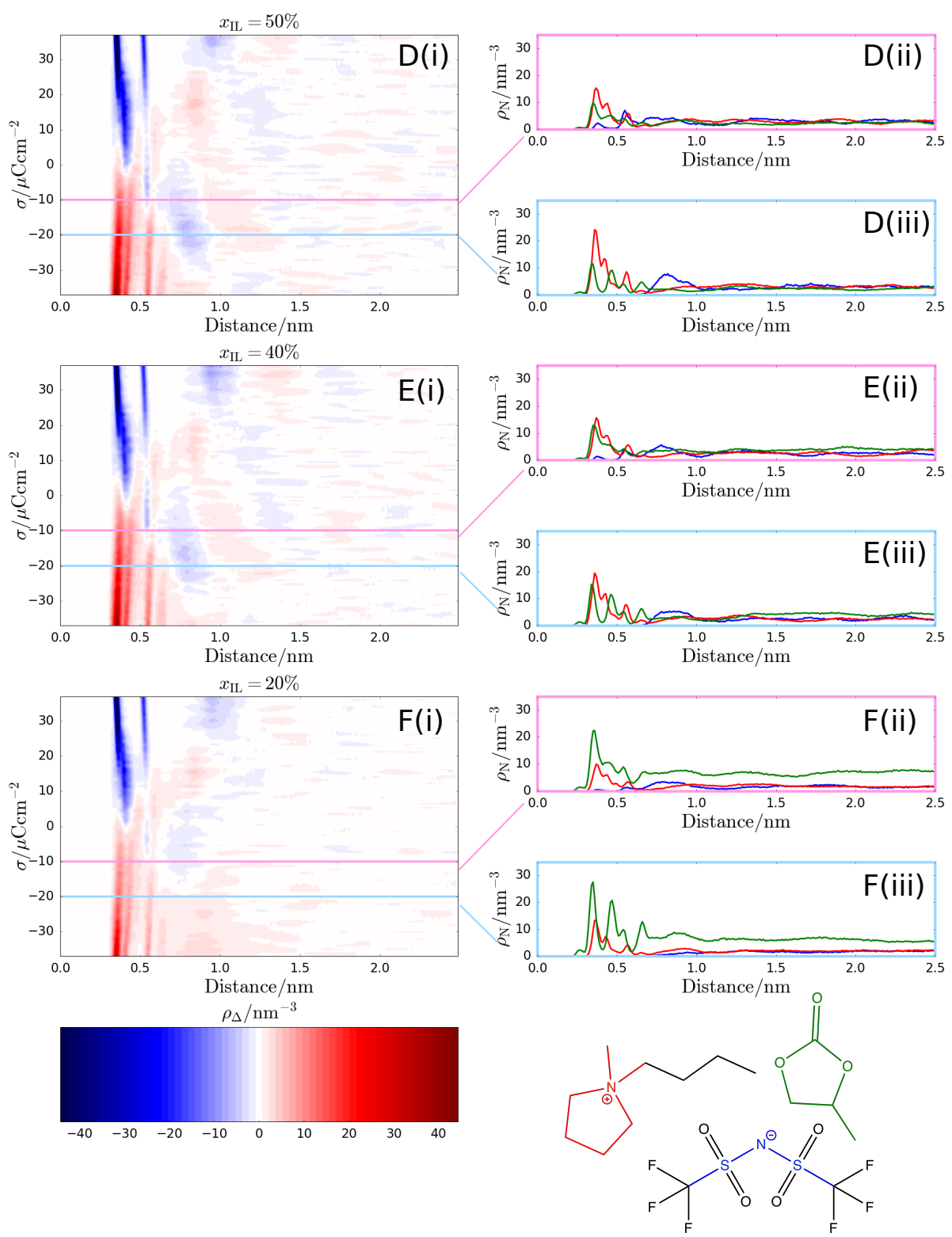

FIG. 3. Left panels: Local difference in number density between cations and anions, $\rho_{\Delta}=\rho_{+}-\rho_{-}$, in mixtures as function of distance from the charged surface and as a function of surface charge density. Six different compositions are studied, with mole fractions ranging from pure ionic liquid, $x_{I L}=100 \mathrm{~mol} \%(\mathrm{~A}(\mathrm{i}))$, to dilute salt in propylene carbonate solvent $x_{I L}=20 \mathrm{~mol} \%(\mathrm{~F}(\mathrm{i}))$. Right panels: density profiles of cation, anion, and solvent as a function of distance from the electrode and at two selected values of surface charge, $-10 \mu \mathrm{Ccm}^{-2}$ (in (ii) for each of A-F) and $-20 \mu \mathrm{Ccm}^{-2}$ (in (iii) for each of A-F). The bottom right structures indicate the regions on each ion that are taken (averaged) to be the 'position' of that ion for calculating local density. 
clear from the alternating regions of excess positive and negative density that our simulations reproduce the charge-oscillatory nanostructure reported in multiple earlier studies of similar systems with pure ionic liquid[26, 27, 58-60]. The position of the first layer of counterions near the surface, at $\sim 0.4 \mathrm{~nm}$, remains static throughout the range of charge densities. The position of the second layer, however, increases from $\sim 0.7 \mathrm{~nm}$ to $\sim 1.0 \mathrm{~nm}$ as the surface charge density increases up to $-36 \mu \mathrm{Ccm}^{-2}$. This appears to be a result of the changing orientation of ions in the first layer, from an in-plane to more perpendicular orientation as their number at the interface increases. The separation between subsequent layers of cations and anions remains largely static at $\sim 0.4 \mathrm{~nm}$ [i.e. a repeat distance of $\sim 0.8 \mathrm{~nm}$ for a cationanion pair] for all values of surface charge. The amplitude of the local charge density in any particular layer is greater with greater magnitude of surface charge; this is exemplified by the comparison between the profiles A(ii) and A(iii) where similar peaks are present but with greater amplitudes at higher negative surface charge density.

As the ionic liquid is diluted with propylene carbonate down to $x_{I L}=80 \mathrm{~mol} \%$ and $x_{I L}=60 \mathrm{~mol} \%$, Figure $3 \mathrm{~B}(\mathrm{i}-\mathrm{iii})$ and $\mathrm{C}(\mathrm{i}-\mathrm{iii})$, the features of the charge density oscillation remain qualitatively similar to that of the pure ionic liquid. Oscillating net charge density is apparent at all surface charges studied, and the peak positions in the ion density profiles remain largely similar. Propylene carbonate appears at significant density within the nm closest to the surface, yet, notably, the absolute positions of the cation and anion peaks are not affected by this and remains static at the $\sim 0.8 \mathrm{~nm}$ repeat distance as in the pure ionic liquid.

Diluting the ionic liquid further down to $x_{I L}=50 \mathrm{~mol} \%$ and $x_{I L}=40 \mathrm{~mol} \%$ (Figure $3 \mathrm{D}$ E) leads to nanostructure that is strongly dependent on the magnitude of surface charge, and varies substantially over the range $0<|\sigma|<36 \mu \mathrm{Ccm}^{-2}$ studied. At $|\sigma| \sim 10-20 \mu \mathrm{Ccm}^{-2}$ the nanostructure is charge-oscillatory (Figure 3 Dii and Eii), as at higher concentrations, however when the charge density is increased to $|\sigma|>30 \mu \mathrm{Ccm}^{-2}$ the decay of ions away from the surface becomes charge-monotonic. (Note that in this context charge-monotonic decay is characterised by excess of counterions over co-ions throughout the interfacial region; this also implies a monotonic decay of potential with distance from the surface.)

When the ionic liquid is diluted with propylene carbonate down to $x_{I L}=20 \mathrm{~mol} \%$ the trend towards charge-monotonic decay in the liquid persists and is apparent at over a substantial range of the surface charge densities studied. For example, inspecting F(iii) it can 
be seen that $\rho_{+}>\rho_{-}$for all distances, and this is the case for all $\sigma>20 \mu \mathrm{Ccm}^{-2}$ and $\sigma<-15 \mu \mathrm{Ccm}^{-2}$. The asymmetry in this effect is likely to arise from the natural asymmetry in the ions themselves, with concomitant impact on packing and screening. At this composition we also note that the propylene carbonate structure becomes strong and, and the absolute number density exceeds that of the ions. (green line in Figure $3 \mathrm{~F}$ ii, iii).

The regions in composition and surface charge space for which we observe chargeoscillatory and charge-monotonic decay are plotted in Figure 4. Several features are apparent: At very low surface charge, where there is little 'screening' taking place and little decay of potential between surface and bulk liquid, the density of cations and anions are relatively uniform at all distances and the manner of decay is not defined. At moderate values of surface charge oscillatory decay is observed for all compositions studied. For the highest concentrations of ionic liquid, up to pure ionic liquid, this charge-oscillatory decay persists even at the highest surface charge densities of $|\sigma| \sim 40 \mu \mathrm{Ccm}^{-2}$. In contrast to this, when the ionic liquid is diluted with polar solvent to compositions of $x_{I L}=60 \mathrm{~mol} \%$ or below, there is a region of charge-monotonic decay. The onset of charge-monotonic decay is at progressively lower surface charge as the ionic liquid content of the mixture decreases.

Finally, we make the comparison between the wavelength transition observed in the SFB experiment and the apparent interfacial nanostructure from MD simulations. In the force measurements the structural transition occurred in the range 30 - $40 \mathrm{~mol} \%$, and the surface charge is estimated (as above) to be $\sim-20 \mu \mathrm{Ccm}^{-2}$. There appear to be two, related, ways of interpreting this in light of the MD simulations. First, inspection of Figure 4 shows that the composition and surface charge of the SFB structural transition appears to coincide with a crossing of the charge-oscillatory to charge-monotonic line in terms of the liquid nanostructure. At salt concentrations above the transition the wavelength of chargeoscillatory decay in the MD simulation and wavelength of the structural force in the SFB experiments are remarkably consistent at $0.8 \mathrm{~nm}$. At concentrations below the cross-over, the structural force wavelength is $0.55 \mathrm{~nm}$ and in the MD simulation solvent oscillations dominate and charge decay becomes monontonic. Secondly, the switch in measured wavelength is likely to be related to the change in dominance from ionic liquid to solvent of the (common) wavelength of the correlation functions. This is seen in the MD simulations as a switch in the dominant component from ionic liquid to solvent that between $x_{I L}=40 \mathrm{~mol} \%$ and $x_{I L}=20 \mathrm{~mol} \%$. That is to say, while both ionic liquid and solvent layering exists within 


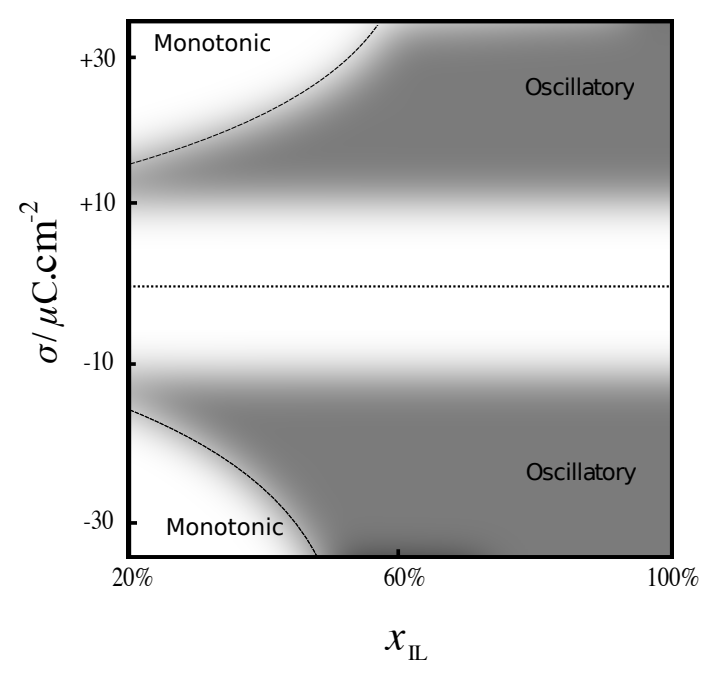

FIG. 4. Interfacial nanostructure diagram indicating the regions in surface charge vs. composition space where the decay of charge density away from the surface is charge-oscillatory or chargemonotonic, determined from MD simulations.

the interfacial nanostructure at all concentrations, the SFB experiment interrogates the lengthscale of the dominant component. That this makes a sharp transition at a fixed mole fraction was the prediction of asymptotic analysis[40] of pair correlation functions in mixtures of large and small particles, borne out in experiments with colloids[41][42], and was the basis of our earlier interpretation[10]. These new MD simulations appear to support and give extra insight into the molecular details underlying the structural transition.

\section{SUMMARY AND CONCLUSION}

By direct comparison of SFB force measurements and MD simulations of the same liquid mixture we have been able to draw the following conclusions. (i) Oscillations in charge density at the electrolyte-electrode interface, well known for pure ionic liquids, are found (in simulations) to persist when the ionic liquid is diluted with a polar solvent over a wide range of $x_{I L}$ and $\sigma$. (ii) The wavelength of charge density oscillations (in the MD simulation) is remarkably consistent, at $0.8 \mathrm{~nm}$, throughout the composition range $100>x_{I L}>40 \mathrm{~mol} \%$. This appears to be consistent with the experimentally determined wavelength of structural forces, which also remains at $\lambda_{o} \sim 0.8 \mathrm{~nm}$, throughout the composition range $100>x_{I L}>$ $40 \mathrm{~mol} \%$. (iii) Diluting the ionic liquid further in the MD simulation leads to two distinct 
changes in structure: a transition to monotonic decay in charge density (this is also surface charge dependent), and strong solvent layering which appears substantially dominant over the ion layering. These features together appear to be consistent with the experimentally determined cross-over to solvent-determined structural forces when $30 \mathrm{~mol} \%>x_{I L}$. (iv) The surface charge on mica immersed in a pure ionic liquid is determined to be approximately $-20 \mu \mathrm{Cm}^{-2}$, allowing direct comparison between force measurements with mica substrates and MD simulations at fixed surface charge. (v) Analysis of the MD simulated structures over a wide range of $x_{I L}$ and $\sigma$ allowed us to map out a phase diagram identifying regions of oscillatory decay of charge density and regions where the charge density decay is monotonic. The structural transition observed in SFB measurements appears to coincide (in terms of surface charge and compostion) with the cross-over from charge-oscillatory to chargemonotonic decay. Asymmetry in the phase diagram around the $\sigma=0$ plane arises from cation-anion asymmetry and therefore highlighting the influence of molecular packing and specific interactions on quantitative details. These effects could not be pursued here where a single ionic liquid was subject of investigation and provoke further work on the effect of ion geometry. Another important avenue for future work includes computer simulation of a more confined electrolyte system, with confinement similar to the SFB experiments to achieve a more direct comparison comparison, and further measurements of the surface charge on mica over a range of electrolyte concentrations and solvent types.

\section{ACKNOWLEDGMENTS}

SC is supported by a Doctoral Training Award from the EPSRC. AMS is supported by a Doctoral Prize from the EPSRC. SP is supported by The Leverhulme Trust (RPG-2015328) and the ERC (under Starting Grant no. 676861, LIQUISWITCH). The authors would like to acknowledge the use of the following computing facilities: the University of Oxford Advanced Research Computing (ARC)[61]; ARCHIE-West High Performance Computing (EPSRC grant no. EP/K000586/1).

[1] R. A. Robinson and R. H. Stokes, Electrolyte solutions (Courier Corporation, 2002). 
[2] L. Suo, O. Borodin, T. Gao, M. Olguin, J. Ho, X. Fan, C. Luo, C. Wang, and K. Xu, Science 350, 938 (2015), http://science.sciencemag.org/content/350/6263/938.full.pdf.

[3] L. Varela, J. Carrete, M. García, L. Gallego, M. Turmine, E. Rilo, and O. Cabeza, Fluid Phase Equilibria 298, 280 (2010).

[4] M. V. Fedorov and A. A. Kornyshev, Chemical Reviews 114, 2978 (2014).

[5] D. Evans and H. Wennerström, The colloidal domain: where physics, chemistry, biology, and technology meet (New York: Wiley-VCH, 1999).

[6] R. G. Horn, D. F. Evans, and B. W. Ninham, The Journal of Physical Chemistry 92, 3531 (1988), http://dx.doi.org/10.1021/j100323a042.

[7] E. K. Humphreys, P. K. Allan, R. J. L. Welbourn, T. G. A. Youngs, A. K. Soper, C. P. Grey, and S. M. Clarke, The Journal of Physical Chemistry B 119, 15320 (2015), pMID: 26513141, http://dx.doi.org/10.1021/acs.jpcb.5b08248.

[8] M. Mezger, R. Roth, H. Schröder, P. Reichert, D. Pontoni, and H. Reichert, J. Chem. Phys. 142, 164707 (2015).

[9] T. Cui, A. Lahiri, T. Carstens, N. Borisenko, G. Pulletikurthi, C. Kuhl, and F. Endres, The Journal of Physical Chemistry C 120, 9341 (2016), http://dx.doi.org/10.1021/acs.jpcc.6b02549.

[10] A. M. Smith, A. A. Lee, and S. Perkin, Phys. Rev. Lett. 118, 096002 (2017).

[11] C. Merlet, M. Salanne, B. Rotenberg, and P. A. Madden, Electrochimica Acta 101, 262 (2013).

[12] J. Vatamanu, M. Vatamanu, O. Borodin, and D. Bedrov, Journal of Physics: Condensed Matter 28, 464002 (2016).

[13] S. Perkin, T. Albrecht, and J. Klein, Physical Chemistry Chemical Physics 12, 1243 (2010).

[14] A. A. Lee, D. Vella, S. Perkin, and A. Goriely, 141, 094904 (2014).

[15] J. Hoth, F. Hausen, M. H. Müser, and R. Bennewitz, Journal of Physics: Condensed Matter 26, 284110 (2014).

[16] M. A. Gebbie, H. A. Dobbs, M. Valtiner, and J. N. Israelachvili, Proceedings of the National Academy of Sciences 112, 7432 (2015).

[17] A. M. Smith, A. A. Lee, and S. Perkin, Journal of Physical Chemistry Letters 7, 2157 (2016).

[18] M. A. Gebbie, A. M. Smith, H. A. Dobbs, A. A. Lee, G. G. Warr, X. Banquy, M. Valtiner, M. W. Rutland, J. N. Israelachvili, S. Perkin, and R. Atkin, 53, 1214 (2017). 
[19] N. Hjalmarsson, R. Atkin, and M. W. Rutland, Chem. Commun. 53, 647 (2017).

[20] A. A. Lee, C. S. Perez-Martinez, A. M. Smith, and S. Perkin, Faraday Discussions (2017).

[21] R. Leote de Carvalho and R. Evans, Molecular Physics 83, 619 (1994).

[22] A. M. Smith, K. R. J. Lovelock, and S. Perkin, Faraday Discuss. 167, 279 (2013).

[23] J. L. Parker and H. K. Christenson, The Journal of Chemical Physics 88, 8013 (1988), http://dx.doi.org/10.1063/1.454260.

[24] S. Páll and B. Hess, Computer Physics Communications 184, 2641 (2013).

[25] M. J. Abraham, T. Murtola, R. Schulz, S. Páll, J. C. Smith, B. Hess, and E. Lindahl, SoftwareX 1-2, 19 (2015).

[26] R. M. Lynden-Bell, A. I. Frolov, and M. V. Fedorov, Phys. Chem. Chem. Phys. 14, 2693 (2012).

[27] V. Ivaništšev, S. O’Connor, and M. V. Fedorov, Electrochem. Commun. 48, 61 (2014).

[28] W. L. Jorgensen, D. S. Maxwell, and J. Tirado-Rives, J. Am. Chem. Soc. 118, 11225-11236 (1996).

[29] J. N. Canongia Lopes and A. A. H. Pádua, J. Phys. Chem. B 108, 16893 (2004).

[30] J. N. Canongia Lopes and A. A. H. Pádua, J. Phys. Chem. B 110, 19586 (2006).

[31] X. You, M. Chaudhari, S. Rempe, and L. R. Pratt, ECS Trans. 69, 107 (2015).

[32] X. You, M. I. Chaudhari, S. B. Rempe, and L. R. Pratt, J. Phys. Chem. B 120, 1849 (2016).

[33] S. Pronk, S. Páll, R. Schulz, P. Larsson, P. Bjelkmar, R. Apostolov, M. R. Shirts, J. C. Smith, P. M. Kasson, D. v. d. Spoel, B. Hess, and E. Lindahl, Bioinformatics 29, 845 (2013).

[34] S. Páll, M. J. Abraham, C. Kutzner, B. Hess, and E. Lindahl, in Solving Software Challenges for Exascale, Vol. 8759, edited by S. Markidis and E. Laure (Springer International Publishing, Cham, 2015) pp. 3-27.

[35] H. J. C. Berendsen, D. van der Spoel, and R. van Drunen, Comput. Phys. Comm. 91, 43-56 (1995).

[36] U. Essmann, L. Perera, M. L. Berkowitz, T. Darden, H. Lee, and L. G. Pedersen, J. Chem. Phys. 103, 8577 (1995).

[37] I.-C. Yeh and M. L. Berkowitz, J. Chem. Phys. 111, 3155 (1999).

[38] H. Christenson and R. Horn, Chemical Physics Letters 98, 45 (1983).

[39] A. M. Smith, K. R. J. Lovelock, N. N. Gosvami, P. Licence, A. Dolan, T. Welton, and S. Perkin, J. Phys. Chem. Lett. 4, 378 (2013). 
[40] C. Grodon, M. Dijkstra, R. Evans, and R. Roth, Journal of Chemical Physics 121, 7869 (2004).

[41] J. Baumgartl, R. P. A. Dullens, M. Dijkstra, R. Roth, and C. Bechinger, Phys. Rev. Lett. 98, 198303 (2007).

[42] A. Statt, R. Pinchaipat, F. Turci, R. Evans, and C. P. Royall, The Journal of Chemical Physics 144, 144506 (2016), http://dx.doi.org/10.1063/1.4945808.

[43] H. K. Christenson and N. H. Thomson, Surface Science Reports 71, 367 (2016).

[44] H. Zhou, M. Rouha, G. Feng, S. S. Lee, H. Docherty, P. Fenter, P. T. Cummings, P. F. Fulvio, S. Dai, J. McDonough, V. Presser, and Y. Gogotsi, ACS Nano 6, 9818 (2012), pMID: 23092400, http://dx.doi.org/10.1021/nn303355b.

[45] R. M. Espinosa-Marzal, A. Arcifa, A. Rossi, and N. D. Spencer, The Journal of Physical Chemistry Letters, The Journal of Physical Chemistry Letters 5, 179 (2014).

[46] R. M. Espinosa-Marzal, A. Arcifa, A. Rossi, and N. D. Spencer, The Journal of Physical Chemistry C, The Journal of Physical Chemistry C 118, 6491 (2014).

[47] H.-W. Cheng, P. Stock, B. Moeremans, T. Baimpos, X. Banquy, F. U. Renner, and M. Valtiner, Advanced Materials Interfaces 2, 1500159 (2015), 1500159.

[48] H. W. Cheng, J. N. Dienemann, P. Stock, C. Merola, Y. J. Chen, and M. Valtiner, Scientific Reports 6, 30058 EP (2016).

[49] S. McDonald, A. Elbourne, G. G. Warr, and R. Atkin, Nanoscale 8, 906 (2016).

[50] P. Campbell, L. Sinnamon, C. Thompson, and D. Walmsley, Surface Science 410, L768 (1998).

[51] A. Deyko, T. Cremer, F. Rietzler, S. Perkin, L. Crowhurst, T. Welton, H.-P. Steinrück, and F. Maier, J. Phys. Chem. C 117, 5101 (2013).

[52] X. Gong, A. Kozbial, and L. Li, Chem. Sci. 6, 3478 (2015).

[53] Z. Wang, H. Li, R. Atkin, and C. Priest, Langmuir 32, 8818 (2016), pMID: 27486675, http://dx.doi.org/10.1021/acs.langmuir.6b01790.

[54] A. A. Lee, D. Vella, S. Perkin, and A. Goriely, The Journal of Physical Chemistry Letters 6, $159(2015)$.

[55] M. T. Alam, M. M. Islam, T. Okajima, and T. Ohsaka, The Journal of Physical Chemistry C 112, 16600 (2008), http://dx.doi.org/10.1021/jp804620m.

[56] J. Wellauer, Zeitschrift für Naturforschung B 68, 1143 (2013). 
[57] R. Tivony and J. Klein, Langmuir 32, 7346 (2016), pMID: 27357375, http://dx.doi.org/10.1021/acs.langmuir.6b01697.

[58] C. Merlet, D. T. Limmer, M. Salanne, R. van Roij, P. A. Madden, D. Chandler, and B. Rotenberg, J. Phys. Chem. C 118, 18291 (2014).

[59] J. Vatamanu, O. Borodin, and G. D. Smith, J. Am. Chem. Soc. 132, 14825 (2010).

[60] J. Vatamanu, L. Cao, O. Borodin, D. Bedrov, and G. D. Smith, J. Phys. Chem. Lett. 2, 2267 (2011).

[61] Richards, 10.5281/zenodo.22558. 